\title{
Origin of very high energy emission in galaxy clusters
}

\author{
V.G. Sinitsyna ${ }^{a}$ and V.Y. Sinitsyna \\ P. N. Lebedev Physical Institute, Russian Academy of Science, Russia
}

\begin{abstract}
The Perseus cluster of galaxies with the central galaxy NGC 1275 is ideally suitable for studying both the physics of relativistic jets from Active Galactic Nuclei and for revealing the feedback role of the central galaxy. We present the results of fifteen-year-long observations of the AGN NGC 1275 at energies $800 \mathrm{GeV}-40 \mathrm{TeV}$ discovered by the SHALON telescope in 1996. The data obtained at very high energies by SHALON, namely the images of the galaxy and its surroundings, and the flux variability indicate that $\mathrm{TeV}$ $\gamma$-ray emission is produced by a number of processes: in particular, part of this emission is generated by relativistic jets in the nucleus of NGC 1275 itself. Unique data on GK Per(Nova 1901) and the IC 310 radio galaxy $\mathrm{TeV} \gamma$-ray emission were obtained with the SHALON experiment for the first time.
\end{abstract}

\section{Introduction}

Clusters of galaxies have long been considered as possible candidates for the sources of $\mathrm{TeV}$ gamma rays emitted by protons and electrons accelerated at large-scale shocks or by a galactic wind or active galactic nuclei (AGNs) [1-9]. The cluster of galaxies in Perseus is one of the beststudied clusters owing to its relative proximity (its distance $\sim 100 \mathrm{Mpc}$ or redshift $\mathrm{z}=0.0179$ ) and brightness. The dominant galaxy in the Perseus cluster is NGC 1275 (Figs. 1, 2, 3).

NGC 1275 has been classified in various ways, for example, as a Seyfert 1.5 galaxy, because broad emission lines were detected in its spectrum at optical wavelengths [10]. However, within the unified model of active galactic nuclei $[11,12]$, it also belongs to the main class of BL Lacertae objects owing to its large and fast flux variability and polarization [13]. It should be noted that there is evidence that the mentioned AGN unification scheme can be even simplified [14].

NGC 1275 is a powerful source of radio and X-ray emission. In the radio band, the object found in NGC 1275, also known as Perseus A and 3C 84, has a powerful and compact core that has been well studied with VLBI [15-17]. NGC 1275 is extremely bright in the radio band and was classified as an FR I radio galaxy; it has a prominent structure that consists of a compact central source and an extended jet $[15,18]$. Having a supermassive black hole (with a mass of $3.4 \times 10^{8} \mathrm{M}_{\odot}$ ) at its center [19], NGC 1275 also exhibits jet precession, which can be interpreted as a possible manifestation of the fact that NGC 1275 is the result of a merger between two galaxies [20]. The radio emission extends to great distances and shows a clear interaction with the gas inside the Perseus cluster of galaxies. ROSAT [8] and, subsequently, Chandra [9] observations revealed cavities in the gas located inside the cluster, whose presence suggests that the jets from $3 \mathrm{C} 84$ sweep up numerous "bubbles" in the atmosphere of the Perseus cluster (Fig. 1).

a e-mail: sinits@sci.lebedev.ru
NGC 1275 surrounded by extended filamentary structures historically aroused great interest owing to both its position at the center of the Perseus cluster and its possible "feedback" role [21]. Evidence for the "feedback" role of NGC 1275 can be obtained from ROSAT and Chandra observations, which reveal shells of hot gas and cavities that spatially coincide with the radio structures (Fig. 3) extending from the central, active part of the AGN. NGC 1275 also arouses interest owing to its close proximity to the Earth at redshift $\mathrm{z}=0.0179$ [22], making it possible to study the physics of relativistic jets.

\section{NGC 1275 viewed in TeV gamma-rays}

Extragalactic sources of very high energy $\gamma$-rays have been searched for in the SHALON experiment from the very beginning of its operation [23,24]. In 1996, observations with the SHALON mirror Cherenkov telescope revealed a new metagalactic source of $\gamma$-ray emission at very high energies $E>800 \mathrm{GeV}[25,26]$ (Figs. 2, 3). The coordinates of an emission source detected in our experiment coincides with the Seyfert galaxy NGC 1275 [25-33]. NGC 1275 was observed by the SHALON telescope for $271.2 \mathrm{~h}$ in different years (from 1996 to 2012) during clear moonless nights at zenith angles from $3^{\circ}$ to $33^{\circ}$. Gamma-ray emission from NGC 1275 was detected by the SHALON telescope at energies above $800 \mathrm{GeV}$ at a $31.4 \sigma$ confidence level determined according to $\mathrm{Li}$ $\&$ Ma [34]. The average integral flux at energies above $800 \mathrm{GeV}$ for NGC 1275 is $(7.8 \pm 0.5) \times 10^{-13} \mathrm{~cm}^{-2} \mathrm{~s}^{-1}$. The $\gamma$-ray energy spectrum in the observed energy range is well described by a power law $F\left(E_{0}>0.8 \mathrm{TeV}\right) \propto E^{k_{\gamma}}$, where $k_{\gamma}=-2.24 \pm 0.09$ (see Fig. 2). Figure 2 shows the source's image at TeV energies observed by the SHALON experiment. The color scale in Fig. 2 right is in units of the excess above the minimum detected signal.

Possible correlations between the emission regions of $\mathrm{TeV} \gamma$-rays and low-energy (radio and X-ray) photons should be established to elucidate the mechanisms of the generation of very high energy emission in the source and

(c) The Authors, published by EDP Sciences. This is an Open Access article distributed under the terms of the Creative Commons Attribution License 4.0 (http://creativecommons.org/licenses/by/4.0/). 

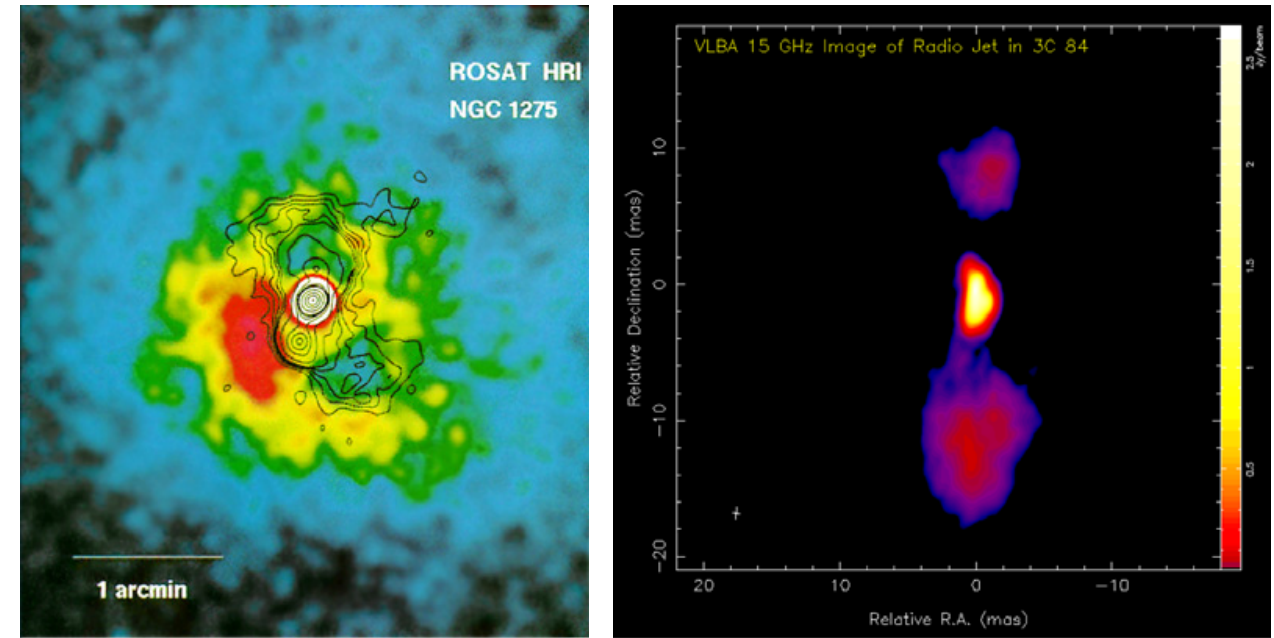

Figure 1. Left: ROSAT X-ray $(0.1-2.4 \mathrm{keV})$ image of NGC 1275 [8]. The contours represent the source's radio structure from VLA radio observations. Right: VLBA $15 \mathrm{GHz}$ image of NGC 1275.
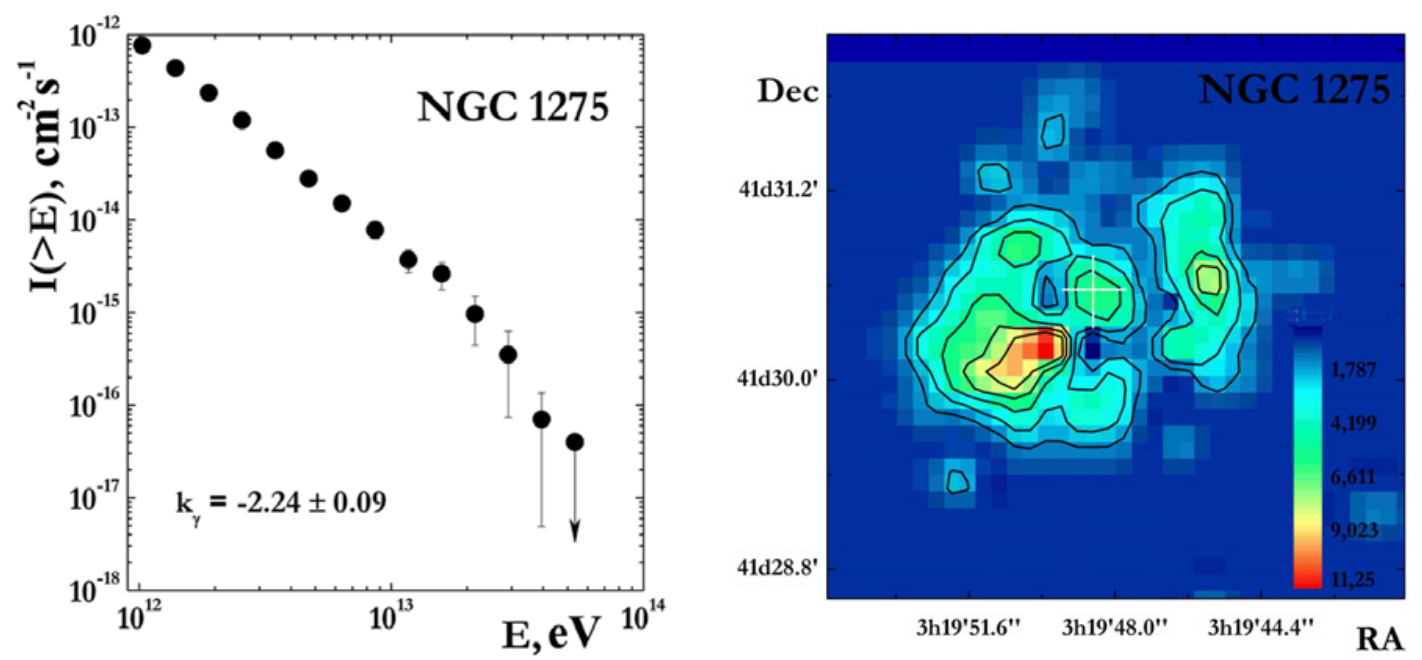

Figure 2. Left: gamma-ray spectrum of NGC 1275 with a power-law index $k_{\gamma}=-2.24 \pm 0.09$. Right: the image of the gamma-ray source NGC 1275 at energies $>0.8 \mathrm{TeV}$ by SHALON.
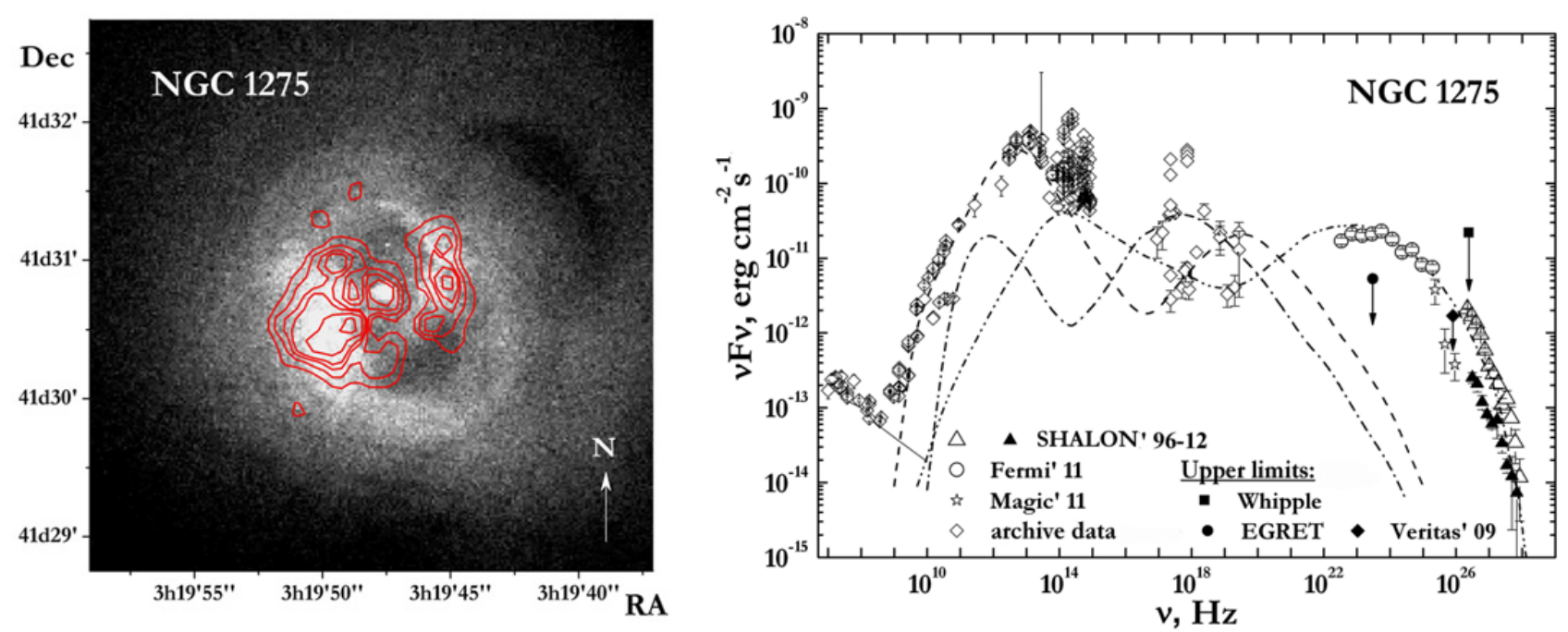

Figure 3. Left: Chandra X-ray (1.5-3.5 keV) image of NGC 1275 [35]; the red contours indicate the SHALON image of NGC 1275 in the energy range $800 \mathrm{GeV}-40 \mathrm{TeV}$. Right: spectral energy distribution of the $\gamma$-ray emission from NGC $1275 . \Delta$ and $\boldsymbol{\Delta}$ represent the data from the SHALON Cherenkov telescope in comparison with experimental data (see [33]). The dashed, dash-dotted, and dash-dotted with two dots curves indicate the spectral energy distributions of NGC 1275 obtained in the CM model [36] and see text below. 
to test the models describing them. Figure 1 left shows a ROSAT X-ray image of NGC 1275; the contours represent the source's radio structure from VLA radio observations and the right is the VLBA $15 \mathrm{GHz}$ image of NGC 1275 with jets (taken from MOJAVE ${ }^{1}$ ). The radio and X-ray emission maxima coincide with AGN NGC 1275 (Fig. 3). But, the X-ray emission disappears almost completely near the bright areas of the radio components located symmetrically relative to the core in the north and the south [8]. We also combined the SHALON-1 (0.8-40 TeV) and Chandra (1.5-3.5 keV X-ray) images. Figure 3 (blackand-white scale) presents a Chandra X-ray $(1.5-3.5 \mathrm{keV})$ image for the central part of the Perseus cluster centered on NGC 1275 with a size of $\sim 5.5$ arcmin [35]. In the $\mathrm{X}$-ray energy range, the core of the Perseus cluster, on the whole, appears as a clear circularly symmetric structure with a distinct maximum on NGC 1275 (Fig. 3).

The clearly seen dimming in X-ray flux, along with the dip NW of the center correlates with the components of the extended double radio structure 3C 84 (Fig. 3). These dips are surrounded by bright (at energies $1.5-3.5 \mathrm{keV}$ ) arc regions from the north and the south. The simplest interpretation is that the intense emission from these rims comes from the shells surrounding the radio lobes [35]. A bright emission spot is also observed to the east.

The emission regions of very high energy $\gamma$-rays observed by SHALON from NGC 1275 have a structure similar to that described above (see [35]) and well correlates with the photon emission regions in the energy range $1.5-3.5 \mathrm{keV}$ (Fig. 3). A correlation of the emission with energies $0.8-40 \mathrm{TeV}$ [28-30] and the X-ray emission in the range $0.3-7 \mathrm{keV}$ [35] was also found. Thus, the $\mathrm{TeV}$ $\gamma$-ray emission recorded by SHALON from NGC 1275 has an extended structure with a distinct core centered at the source's position.

To analyze the emission related to this core, we additionally identified the emission component corresponding to the central region of NGC 1275 with a size of $32^{\prime \prime}$. The emission from the central region of NGC 1275 was detected at energies above $0.8 \mathrm{TeV}$ at a $13.5 \sigma$ confidence level determined by the $\mathrm{Li}$ \& Ma method [34] with an average integral flux $(3.26 \pm 0.30) \times 10^{-13} \mathrm{~cm}^{-2} \mathrm{~s}^{-1}$. The $\gamma$-ray energy spectrum of the central component in the entire energy range from 0.8 to $40 \mathrm{TeV}$ is described by a power law with an exponential cutoff, $I\left(>E_{\gamma}\right)=(2.92 \pm 0.11) \times 10^{-13} \times$ $E^{-1.55 \pm 0.10} \times \exp \left(-E_{\gamma} / 10 \mathrm{TeV}\right) \mathrm{cm}^{-2} \mathrm{~s}^{-1}$. The SHALON spectrum corresponding to the emission from the central region of NGC 1275 is represented in Fig. 3 with the black triangles.

NGC 1275 was detected at high energies of $100 \mathrm{MeV}-300 \mathrm{GeV}$, by the Fermi LAT experiment [37]. Recently, the AGN NGC 1275 was also recorded by the MAGIC ground-based mirror Cherenkov telescope at energies above $100 \mathrm{GeV}$ in the 2010-2011 observations [38].

Figure 3 compares the integral $\gamma$-ray spectrum of NGC $1275(\triangle)$ and its central region $(\Delta)$ obtained from SHALON data (1996-2012) [33] with the Fermi LAT (2009-2011) [37] and MAGIC (2010-2011) [38] experimental data.

\footnotetext{
${ }^{1}$ http://www . physics.purdue.edu/astro/MOJAVE/
}

\section{Flaring activity of NGC 1275 in high and very high energies}

The revealing correlations between the emissions in different energy ranges, comparing the emission regions, and, in particular, the detection of the flux changes remains necessary, because it makes it possible to judge the nature of the source, its evolution, and the emission generation mechanisms in various objects.

The observed $\gamma$-ray flux variations do not, on average, exceed $20 \%$ of $(7.8 \pm 0.5) \times 10^{-13} \mathrm{~cm}^{-2} \mathrm{~s}^{-1}$. The SHALON mirror Cherenkov telescope has detected three short-time (within five days) increases and one decrease of the very high energy $\gamma$-ray flux in the entire time of observations of NGC 1275. Given these variations, the flux decrease below the average was recorded in 1999 and the integral flux was $(4.7 \pm 1.3) \times 10^{-13} \mathrm{~cm}^{-2} \mathrm{~s}^{-1}$. The increases were detected in late Jan. 2001, late Nov. early Dec. 2005, and late Oct. 2009. The fluxes in these periods were $(21.2 \pm 7.5) \times 10^{-13},(35.5 \pm 12.4) \times 10^{-13}$, and $(23.4 \pm 4.5) \times 10^{-13} \mathrm{~cm}^{-2} \mathrm{~s}^{-1}$, respectively. The duration of the flux increase in October 2009 was 3 days. No duration of intervals of flux increase were found in 2001 and 2005, because the observations were interrupted due to weather conditions in both cases.

To reveal possible correlations of the emissions in various energy ranges, including those at high and very high energies, we compared the NGC $1275 \gamma$-ray fluxes from SHALON in the periods when the observations were simultaneous with the ones by the Fermi LAT experiment. The published Fermi LAT data were obtained from August 4, 2008, to September 30, 2010 [37]. SHALON observations of NGC 1275 were performed in November 2008 with a break for the Moon's time, October 2009, and mid-November-early December 2010. In this time, only one $\gamma$-ray flux increase to $(23.4 \pm 4.5) \times 10^{-13} \mathrm{~cm}^{-2} \mathrm{~s}^{-1}$ was detected in the period of October 18-20, 2009. These periods of SHALON observations do not coincide with the times of the main flares observed at Fermi LAT [37]. A slight local flux increase can be seen in the period of mid-October 2009 [37], which corresponds to the abovementioned $\gamma$-ray flux increase observed by SHALON.

\section{Sources during observations of NGC 1275}

Additionally an analysis of the region around NGC 1275 was performed in order to search for $\mathrm{TeV}$ gamma-ray sources in the SHALON telescope field of view during the observation of the Perseus Cluster.

\subsection{SN2006 gy}

The flux increase was detected from the region NGC 1275 in autumn 2006. The detailed analysis of $\gamma$-shower direction turned out to be the detection of a metagalactic object. This object was identified with the supernova SN2006 gy (see [39]) that is about 10 minutes away from NGC 1275 (see details in [40]).

Observations had been made in 2006 September, October, November, December and then during the winter of 2007. No flux increase was found in the September observations. In the flare, observed on October 22, the flux increased 6 times from the NGC 1275 average flux 

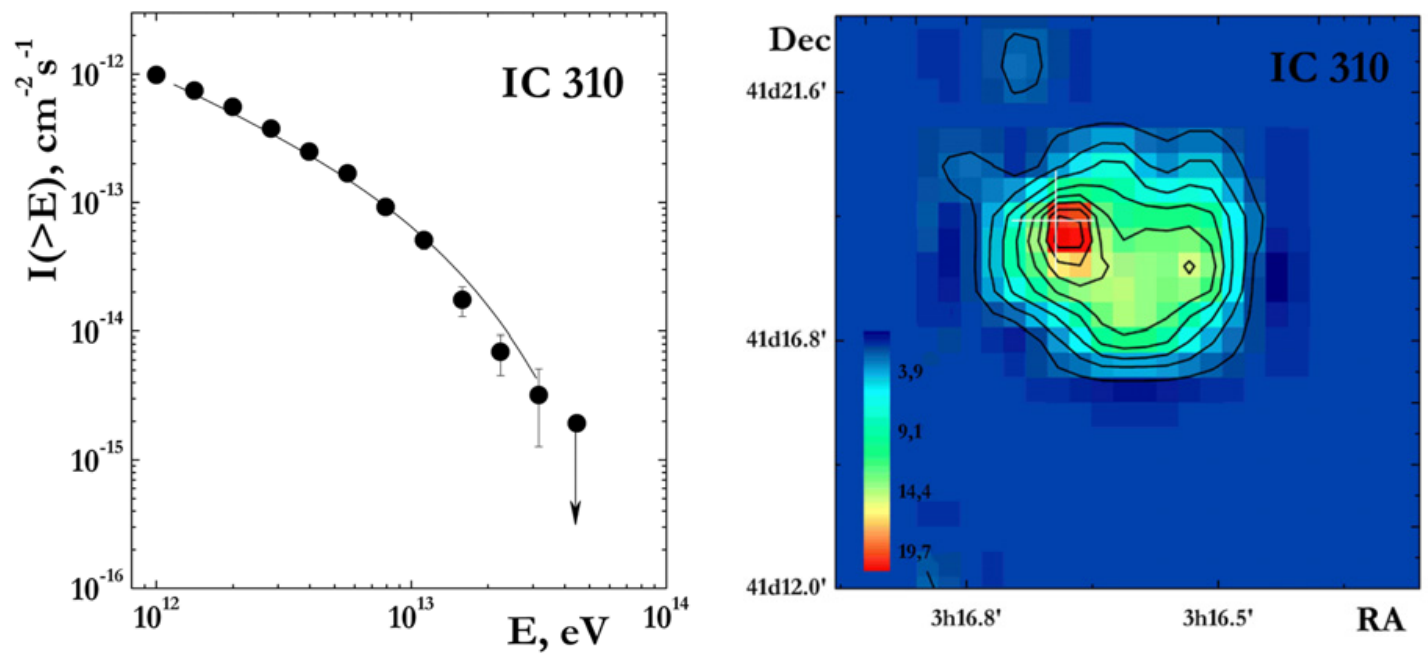

Figure 4. Left: gamma-ray spectrum of IC 310 by SHALON. Right: the image of the gamma-ray source IC 310 at energies $>0.8 \mathrm{TeV}$ by SHALON.

and stayed at this level throughout the October moonless period. The integral $\gamma$-ray flux for SN 2006gy was found to be $(3.71 \pm 0.65) \times 10^{-12} \mathrm{~cm}^{-2} \mathrm{~s}^{-1}[40,41]$ at energies of $>0.8 \mathrm{TeV}$. Follow-up observations at the end of November showed that the flux of SN2006 gy had dropped to a level of about $(0.69 \pm 0.17) \times 10^{-12} \mathrm{~cm}^{-2} \mathrm{~s}^{-1}$ and was constant during the November, December period. The results of observation analysis of 2007 have not revealed any TeV gamma-ray emission from the region of SN 2006gy. So, the explosion of extragalactic supernova was observed at $\mathrm{TeV}$ energies for the first time with the SHALON Cherenkov telescope.

\subsection{GK Per}

During the observations of NGC 1275 the SHALON field of view contains the source of non-thermal radio and X-ray emission GK Per (Nova 1901) of classical nova type as it is located at $\sim 3^{\circ} \mathrm{SW}$ from NGC 1275. So due to the large telescopic field of view $\left(\geq 8^{\circ}\right)$ all observations of NGC 1275 are naturally followed by the tracking of GK Per. These long-term observations of the Perseus Cluster revealed $\gamma$-ray emission from a nearby object -GK Per (see details in [42]). The $\gamma$-ray source associated with GK Per was detected above $800 \mathrm{GeV}$ with average $\gamma$-flux $I_{G K \text { Per }}(>0.8 \mathrm{TeV})=(2,9 \pm 1,3) \times 10^{-13} \mathrm{~cm}^{-2} \mathrm{~s}^{-1}$ with a statistical significance [34] of $9.2 \sigma$. The analysis of $\gamma$-ray shower arrival direction revealed the main $\mathrm{TeV}$-emission region coinciding with the position of the central source of GK Per and the weak emission of shell, that is also observed in X-ray by Chandra [43].

The TeV $\gamma$-ray emission of classical nova GK Per, that could be a shell-type supernova remnant in the early evolution stage, was detected for the first time by SHALON. Also, very high energy $\gamma$-rays from the shell of GK Per, visible in X-rays, were detected with SHALON experiment for the first time.

\subsection{IC 310}

The radio galaxy IC 310 (redshift $\mathrm{z}=0.0189$ ) is located in the Perseus Cluster at $0.6^{\circ}$ (about $1 \mathrm{Mpc}$ ) from the cluster's central galaxy, NGC 1275. It is one of the brightest objects of this Cluster of galaxies at radio frequencies and at $\mathrm{X}$-ray energies. It has been detected in the high energy $\gamma$-ray band above $30 \mathrm{GeV}$ with Fermi LAT [44] and above $260 \mathrm{GeV}$ with the MAGIC telescopes [45].

The radio morphology of such types of galaxies consists of a bright "head", which is located at the core of the galaxy, and the "tail" of a radio lobe pointing away from the center of the cluster (Fig. 5). The jet direction is determined by the motion of galaxy through the medium of the cluster. In the case of IC 310, the radio lobe with a length of about $400 \mathrm{kpc}$ extends in the direction parallel to the line connecting IC 310 with the center of the Perseus cluster.

A recent investigation of the radio structure of IC 310 by VLBA shows that the "head" contains a parsec-scale core-jet structure oriented in the same direction as the kiloparsec structure of the "tail". That means that there is no evidence of an interaction with the intracluster medium determining the direction of the "tail" [46]. In X-rays IC 310 looks as a point source at the position of the radio "head" [47].

IC 310 as a source accompanying NGC 1275 was observed with SHALON telescope between 1996 and 2012 for a total of 157 hours. The $\gamma$-ray source associated with IC 310 was detected above $0.8 \mathrm{TeV}$ by SHALON with a statistical significance $17.2 \sigma$ determined by the $\mathrm{Li} \&$ Ma method [34]. The signal significance for this source is less then one for the source with a similar flux and spectrum index obtained in the same observation hours because of smaller collection field of view relative to the standard procedure of the SHALON experiment [33]. Corrections for the effective field of view were made to calculate the source flux and energy spectrum. The average integral flux at energies above $0.8 \mathrm{TeV}$ for IC 310 is $(0.89 \pm 0.09) \times 10^{-13} \mathrm{~cm}^{-2} \mathrm{~s}^{-1}$ (Fig. 4). Taking into account the proximity to a nearby source NGC 1275 , we made the observation data procession first associated with NGC 1275 and then with IC 310. We found that 5\% of showers are common for both sources. After the detailed analysis of the arrival direction of these showers and angular distance [33], less than 2\% of NGC 1275 showers were recognized to be IC 310 showers. This didn't change the average flux of NGC 1275 [33]. The energy spectrum 

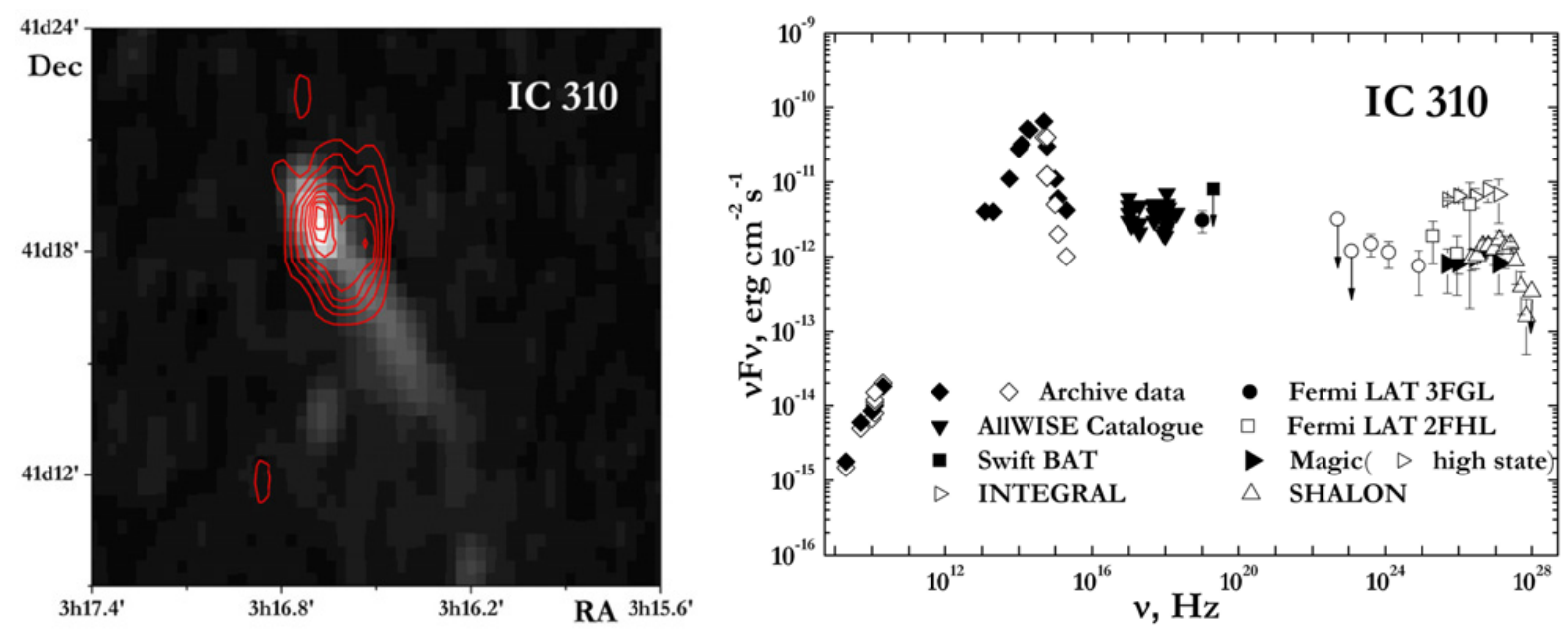

Figure 5. Left: radio image of IC 310 from WENSS sky survey; the red contours indicate the SHALON image of IC 310 in the energy range $800 \mathrm{GeV}-50 \mathrm{TeV}$. Right: Spectral energy distribution of the $\gamma$-ray emission from IC310. $\triangle$ represent the data from the SHALON Cherenkov telescope.

of $\gamma$-rays in the observed energy region from 0.8 to $40 \mathrm{TeV}$ is well described by a hard power law with an exponential cutoff $I\left(>E_{\gamma}\right)=(0.89 \pm 0.09) \times 10^{-12} \times E^{-0.84 \pm 0.10} \times$ $\exp \left(-E_{\gamma} / 12.5 \mathrm{TeV}\right) \mathrm{cm}^{-2} \mathrm{~s}^{-1}$ (see Fig. 4). The spectral energy distribution and detailed images of IC 310 are shown in Fig. 5.

The main $\mathrm{TeV} \gamma$-ray emission region was revealed in observations of IC 310 corresponding to the core of the galaxy. It coincides with the position of the "head" of the radio structure of IC 310. Figure 5, left (black-and-white scale) shows a radio image of the IC 310 galaxy from the WENSS survey [48] (SkyView interface ${ }^{2}$ ), red lines show the TeV structure of IC 310 by SHALON.

\section{NGC 1275 as a point and extended source}

As has been pointed out above, the Perseus cluster of galaxies with the central galaxy NGC 1275 is ideally suitable for studying both the physics of relativistic jets from AGNs and for revealing the feedback role of the central galaxy. Evidence for the latter was obtained in ROSAT and Chandra observations at low energies, from which shells of hot gas and cavities that spatially coincide with the radio structures originating in the central, active part of the AGN can be seen (Fig. 1 and $[8,9,35,49]$ ). The observational data for NGC 1275 at energies $800 \mathrm{GeV}-40 \mathrm{TeV}$, namely the images of the galaxy and its surroundings (Figs. 2, 3), as well as the flux variations suggest that the $\mathrm{TeV}$ gamma-ray emission in these regions is produced by a number of processes.

The extended structure around NGC 1275 (Fig. 3) that spatially coincides with the $\mathrm{X}$-ray emission regions (Fig. 3) can be produced by mechanisms related to the generation of an X-ray structure $[9,35,49]$. The brightness distribution of the X-ray emission and the observed $\mathrm{TeV}$ emission shows a sharp increase in intensity right outside the bubbles blown by the central black hole and visible in the radio band. This suggests that the X-ray-generating particles are swept up from the region of the radio lobes

$\overline{2 \text { http://skyview.gsfc.nasa.gov/ }}$ under the pressure of cosmic rays and magnetic fields generated in the jets at the center of NGC $1275[9,49]$. The structures visible in $\mathrm{TeV} \gamma$-rays are formed through the interaction of very high energy cosmic rays with the gas inside the Perseus cluster and interstellar gas heating at the boundary of the bubbles blown by the central black hole in NGC 1275.

The presence of emission in the energy range $1-40 \mathrm{TeV}$ from a central region of $\sim 32^{\prime \prime}$ in size around the nucleus of NGC 1275 (see Fig. 3, the black triangles) and the shorttime flux variability [33] point to the origin of the very high energy emission as a result of the generation of jets ejected by the central supermassive black hole of NGC 1275 .

The multifrequency spectral energy distribution for the nucleus of NGC 1275, up to high and very high energies, was described in the CM model [36] and is a composition of the components of inverse Compton scattering of the intrinsic synchrotron radiation from relativistic electrons (synchrotron self-Compton) of three separate plasma blobs ejected from the inner regions of the NGC 1275 nucleus (Fig. 3, the dashed, dash-dotted, and dash-dotted-dotted curves). The available Fermi LAT data at high energies and the SHALON observations at very high energies in a region < 32" around NGC 1275 are described in terms of this model with one of the components producing synchrotron self-Compton emission of the relativistic jets from the nucleus itself (Fig. 3, the dash-dotted-dotted curve).

\section{Conclusion}

We present the results of fifteen-year-long observations of the AGN NGC 1275 at energies $800 \mathrm{GeV}-40 \mathrm{TeV}$ discovered by the SHALON telescope in 1996. Having analyzed the SHALON data, we have determined characteristics of NGC 1275 such as the spectral energy distributions and images at energies $>800 \mathrm{GeV}$ for the first time. The emission regions of very high energy gamma-rays observed by SHALON from NGC 1275 well correlates with the photon emission regions viewed in $\mathrm{X}$-rays by Chandra. Thus, the $\mathrm{TeV}$ gamma-ray emission recorded by SHALON has an extended structure with a distinct core centered at the source's position. To analyze 
the emission related to this core, we additionally identified the emission component corresponding to the central region of NGC 1275, and obtained the spectral energy distribution of this component as a result. Variations of $\mathrm{TeV}$ gamma-ray fluxes were also found. The data obtained at very high energies, namely the images of the galaxy and its surroundings, and the flux variability indicate that the TeV gamma-ray emission is generated by a number of processes: in particular, part of this emission is generated by relativistic jets in the nucleus of NGC 1275 itself. The presence of an extended structure around NGC 1275 is evidence of the interaction of cosmic rays and magnetic fields generated in the jets at the galactic center with the gas of the Perseus cluster.

\section{References}

[1] B. Dennison, Astrophys. J. 239, L93 (1980)

[2] B.P. Houston et al., J. Phys. G: Nucl. Phys. 10, L147 (1984)

[3] S. Colafrancesco et al., Astropart. Phys. 9, 227 (1998)

[4] C.L. Sarazin Astrophys. J. 520, 529 (1999)

[5] F.T. Miniati et al., Astrophys. J. 62, 233 (2001)

[6] A.N. Timokhin et al., Astron. Astrophys. 417, 391 (2004)

[7] S. Colafrancesco, P. Marchegiani, Astron. Astrophys. 502, 711 (2009)

[8] H. Böhringer et al., Mon. Not. R. Astron. Soc. 264, L25 (1993)

[9] A.C. Fabian et al., Mon. Not. R. Astron. Soc. 366, 417 (2006)

[10] M.P. Veron-Cetty, P. Veron. Quasars and Active Galactic Nuclei, 8th ed., ESO Sci. Rep. No. 18 (ESO, 1998); ADC/CDS Catalogue No.7207 (1998)

[11] B.L. Fanaroff, J.M. Riley, Mon. Not. R. Astron. Soc. 167, 31P (1974)

[12] C.M. Urry, P. Padovani, Publ. Astron. Soc. Pacif. 54, 215 (1995)

[13] J.R.P. Angel, H.S. Stockman, Ann. Rev. Astron. Astrophys. 18, 321 (1980)

[14] P. Kharb et al., Astrophys. J. 710, 764K (2010)

[15] R.C. Vermeulen, et al., Astrophys.J. 430, L41 (1994)

[16] G.B. Taylor, R.C. Vermeulen, Astrophys. J. 457, L69 (1996)

[17] K. Asada et al., Publ. Astron. Soc. Jpn. 58, 261 (2006)

[18] K. Asada et al., Astron. Soc. Pacif. Conf. 402, 91A (2009)

[19] R.J. Wilman et al., Mon. Not. R. Astron. Soc. 359, $755(2005)$

[20] F.K. Liu, X. Chen, Astrophys. J. 671, 1272 (2007)
[21] J.S. Gallagher, Astron. Nachr. 220, 1040G (2009)

[22] M. Strauss et al., Astrophys. J. Suppl. Ser. 83, 29S (1992)

[23] V.G. Sinitsyna, Proc. of the Workshop, Towards a Major Atmospheric Cherenkov Detector IV, Padova, Italy, ed. M. Cresti (Univ. of Padova, Padova, Italy, 1995), p. 133

[24] V.G. Sinitsyna, Nuovo Cim. 19C, 965 (1996)

[25] V.G. Sinitsyna, Proc. of the Workshop, Towards a Major Atmospheric Cherenkov Detector V, Kruger Park, South Africa, ed O. De Jager (Westprint, Potchefstroom, 1997), p. 136

[26] V.G. Sinitsyna, AIP (Conf. Proc.) 515, 293 (2000)

[27] V.G. Sinitsyna, Rad. Phys. Chem. 75, 880 (2006)

[28] V.G. Sinitsyna et al., Nucl. Phys. B (Proc. Suppl.) 122, 409 (2003)

[29] V.G. Sinitsyna et al., Bull. Russ. Acad. Sci. 71, 906 (2007)

[30] V.G. Sinitsyna et al., Nucl. Phys. B (Proc. Suppl.) 196, 442 (2009)

[31] S.I. Nikolsky, V.G. Sinitsyna, Phys. At. Nucl. 67, 1900 (2004)

[32] V.G. Sinitsyna et al., Nucl. Phys. B (Proc. Suppl.) 75A, 352 (1999)

[33] V.G. Sinitsyna, V.Y. Sinitsyna, Astron. Lett. 40(2-3), 91 (2014)

[34] T.-P. Li and Y.-Q. Ma, Astrophys. J. 272, 317 (1983)

[35] A.C. Fabian et al., Mon. Not. R. Astron. Soc. 318, L65 (2000)

[36] S. Colafrancesco et al., Astron. Astrophys. 519, A82 (2010)

[37] A.M. Brown, J. Adams, Mon. Not. R. Astron. Soc. 413, 2785 (2011)

[38] J. Aléksic et al., Astron. Astrophys. 539, L2 (2012)

[39] E.O. Ofek et al., arXiv: astro-ph/0612408v1

[40] V.G. Sinitsyna, V.Y. Sinitsyna, EPJ Web of Conferences 52, 10005 (2013)

[41] V.G. Sinitsyna et al., 2011, "Cosmic Rays For Particle And Astroparticle Physics" Proc. 12th ICATPP (Eds. S. Giani, C. Leroy, and P.G. Rancoita; Singapore: World Scientific) 6, 296 (2011)

[42] V.G. Sinitsyna, V.Y. Sinitsyna, J. Phys.: Conf. Ser. 718, 052046 (2016)

[43] S. Balman, Astrophys. J. 627, 933 (2005)

[44] A. Neronov et al., Astron. Astrophys. 519, L6 (2010)

[45] J. Aleksić et al., Astrophys. J. Lett. 723(2), L207 (2010)

[46] M. Kadler et al., Astron. Astrophys. 538, L1 (2012)

[47] K. Sato et al., PASJ 57, 743 (2005)

[48] R.B. Rengelink et al., A\&AS 124, 259 (1997)

[49] E. Churazov et al., A\&A 356, 788 (2000) 